\title{
Podes me ajudar? Apoiando a formação de pares em sistemas de ajuda em pares através de técnicas de recomendação
}

\author{
Luana Müller, Milene Selbach Silveira \\ Programa de Pós-Graduação em Ciência da Computação - Faculdade de Informática \\ Pontifícia Universidade do Rio Grande do Sul \\ Porto Alegre - RS - Brasil \\ luana.muller@acad.pucrs.br, milene.silveira@pucrs.br
}

\begin{abstract}
Recommender systems have been disseminated by helping users to find products or adequate information to their profiles in the immensity of options available on the Internet nowadays. This research aims to integrate techniques used by these systems to peer help systems in order to support the formation of pairs using social matching (recommendation of people), and in this way trying to help users to solve their questions. The proposed process to pair formation was developed in a peer help system prototype focused in collaborative learning environments that allow analyzing the formation of these pairs, and thus obtaining information about the effectiveness of its use.
\end{abstract}

Resumo. Sistemas de recomendação tem se disseminado ajudando usuários a encontrar produtos ou informações adequados ao seu perfil em meio à imensidão de opções disponíveis na Internet hoje em dia. A presente pesquisa visa integrar as técnicas usadas por estes sistemas aos sistemas de ajuda em pares, visando apoiar a formação destes pares usando combinação social (recomendação de pessoas), e tentando, assim, auxiliar os usuários para que estes consigam sanar suas dúvidas. O processo proposto para formação de pares foi implementado em um protótipo de sistema de ajuda focado em ambientes colaborativo de ensino, permitindo analisar a formação destes pares e assim obter informações a respeito da efetividade de seu uso.

\section{Introdução}

Criados para auxiliar os usuários a adquirirem os conhecimentos requeridos para que pudessem utilizar os sistemas que necessitam, os sistemas de ajuda on-line [Joseph 1980] enfrentam a resistência dos usuários quanto a seu uso, pois as informações por eles fornecidas muitas vezes não podem ser aplicadas a problemas específicos de determinado usuário [Vouligny e Robert 2005]. Na tentativa de apoiar os usuários na resolução destes problemas, os sistemas de ajuda em pares surgiram, visando, através da interação entre usuários, incentivar que a ajuda seja oferecida pelos próprios usuários da aplicação, complementando desta forma a ajuda oferecida pelo sistema de ajuda tradicional [Kumar 2004][Leite e Silveira 2010].

E, no intuito de apoiar a formação destes pares é que esta pesquisa se situa, e, para isto foram utilizadas técnicas provenientes dos sistemas de recomendação. Estes sistemas trazem a ideia de que usuários que compartilham do mesmo interesse no passado, terão preferências similares no futuro, podendo com base nessas informações, 
recomendar itens a eles. Estes sistemas têm sido amplamente utilizados em diversos domínios recomendando produtos, conteúdos, serviços ou pessoas [Torres 2004].

No presente trabalho, aliaram-se, então, técnicas de recomendação ao processo de seleção de pares de um sistema de ajuda em pares para o ambiente colaborativo de ensino Moodle, promovendo uma combinação social [Motta 2011] através da qual esses pares formados irão interagir na tentativa de solucionar dúvidas que existam em relação à utilização do ambiente.

O sistema de ajuda proposto foi inicialmente focado a usuários com perfil de professor neste ambiente. Esta escolha foi feita por ter sido verificado que usuários que possuem este perfil tendem a ter mais dúvidas sobre a utilização do ambiente devido a grande quantidade de recursos e atividades que costumam necessitar ser configuradas uma a uma por eles [Baqueta et al. 2011].

As seções a seguir apresentarão o referencial teórico que embasa este trabalho, seguido do detalhamento e resultados do processo de recomendação proposto, assim como, uma discussão a respeito destes resultados. E, por fim são apresentadas as considerações finais e referências bibliográficas utilizadas.

\section{Sistemas de Recomendação}

Sistemas de recomendação são classificados como ferramentas de software e técnicas que provem aos usuários sugestões de itens, sendo "itens" o termo utilizado para definir o que esta sendo recomendado pelo sistema ao usuário [Ricci et al. 2010].

Esses sistemas são utilizados para identificar os usuários, salvar suas preferências e recomendar a eles itens, itens estes que podem ser produtos, serviços, conteúdo e, até mesmo, pessoas ${ }^{1}$, de acordo com as suas necessidades e interesses [Barcellos et al. 2007][ Motta et al. 2011].

Um dos maiores desafios existentes na construção dos sistemas de recomendação esta em combinar a expectativa do usuário e os itens a serem recomendados a ele. Esses sistemas precisam ser capazes de identificar as preferencias dos usuários e sugerir itens relevantes para cada um deles, de forma personalizada, com base no seu comportamento de navegação, consultas e compras feitas [Cazella, Reategui e Nunes 2010].

\section{Sistemas de Ajuda}

Os sistemas de ajuda on-line foram criados para ser uma alternativa que ajudasse usuários a se tornarem autossuficientes e menos dependentes de assistência pessoal [Joseph 1980]. Desde então, eles têm sido desenvolvidos tentando atender as necessidades dos usuários, e seu design e desenvolvimento tem evoluído junto com as aplicações e tecnologias que eles assistem.

Sobre sua utilização, usuários geralmente recorrem a eles por duas razões: porque estão confusos sobre determinada funcionalidade ou porque eles precisam

\footnotetext{
${ }^{1}$ Sistemas de recomendação que tem pessoas como seu item de recomendação são também chamados Sistemas de Combinação Social.
} 
encontrar determinada funcionalidade [Spool e Scalon 1996]. No entanto, pesquisas mostram que esses sistemas não são usados por usuários que necessitam assistência porque os sistemas de ajuda fornecem uma informação genérica e que não pode ser aplicada a determinadas situações [Vouligny e Robert 2005].

Com base nisso, os sistemas de ajuda em pares surgiram para adicionar uma nova abordagem aos sistemas de ajuda tradicionais, visando encorajar e qualificar seu uso [Leite e Silveira 2010]. Os sistemas de ajuda em pares representam uma rede integrada de usuários, possuindo conhecimento sobre eles, o qual é usado para promover uma interação entre pares de usuários para tentar resolver suas dúvidas adequadamente [Kumar 2004].

Neste âmbito, Leite e Silveira (2010) propõem uma arquitetura de sistemas de ajuda em pares para ambientes virtuais de ensino e aprendizagem, chamada PHAVEA. Nesta abordagem, os pares são gerados considerando o nível do usuário, nível este calculado com base nas avaliações obtidas pelas respostas por ele dadas aos demandantes, não sendo consideradas informações a respeito do perfil do usuário para a formação destes pares.

\section{Aplicando técnicas de recomendação em sistemas de ajuda em pares}

Sistemas de ajuda não são frequentemente utilizados por usuários, e uma das razões é o fato de as informações contidas nesses sistemas não poderem ser aplicadas a uma questão mais específica ou contextualizada do usuário. Neste caso, o auxílio de outros usuários acaba sendo a forma mais procurada na hora de esclarecer uma dúvida [Leite e Silveira 2010, Vouligny e Robert 2005], o que pode ser abordado com o paradigma de pesquisa chamado paradigma da vila. Em uma vila, a informação é disseminada socialmente, e a principal forma de construir o conhecimento consiste em encontrar a pessoa certa para responder uma questão, diferentemente do paradigma tradicional, no qual a recuperação de informação ocorre como em uma biblioteca, utilizando palavraschaves para fazer as buscas [Damon e Kamvar 2010]. Considerando os princípios do paradigma da vila, os sistemas de ajuda em pares ajudam a prover a interação entre pares de usuários, para ajuda-los a encontrar ajuda através de outro usuário e tentar com isso complementar a ajuda oferecida pelo sistema de ajuda [Müller e Silveira 2012].

Uma das etapas realizadas pelos sistemas de ajuda em pares consiste em localizar e sugerir ao usuário uma pessoa para ajuda-lo. Baseado nisso, é aqui proposto integrar técnicas de recomendação aos sistemas de ajuda em pares para tentar com isso apoiar o processo de formação de pares de usuários e, assim, ajudar o usuário a encontrar a pessoa certa (ou mais adequada) para responder a sua questão, tentando oferecer, a estes usuários, uma melhor experiência com o sistema de ajuda e também com o ambiente que esta sendo utilizado.

O sistema de ajuda em pares proposto foi aplicado a ambientes colaborativos de ensino. A principal razão por decidir usar estes ambientes como cenário para o processo de ajuda em pares é por este se mostrar um contexto no qual os usuários precisam frequentemente lidar com dúvidas em relação ao seu uso. Com o crescente aumento do uso destes ambientes e diversidade de perfis de usuários que os utilizam, é cada vez mais importante que eles sejam de simples e fácil utilização. Assim, é importante que os usuários tenham facilidade e sintam-se à vontade neste ambiente. No entanto é visto que 
tanto a essa diversidade de perfis de usuários quanto de recursos e atividades configuráveis que tais ambientes disponibilizam faz com que os usuários necessitem de ajuda constantemente [Baqueta et al. 2011].

\subsection{Processo de recomendação da ajuda}

Sendo o objetivo da presente pesquisa, auxiliar usuários a sanar suas dúvidas sobre o uso do ambiente, serão usados no processo de recomendação proposto os critérios tempo de uso do ambiente, conhecimento técnico e receptividade, apontados por Müller e Silveira (2012) como critérios que podem ser usados quando o objetivo é auxiliar usuários sobre dúvidas do ambiente. Os critérios serão usados da seguinte forma:

1. Tempo de uso do ambiente: critério usado através da medição do tempo de uso do ambiente de cada usuário, encaminhando as dúvidas aos usuários que possuem mais tempo.

2. Conhecimento técnico e receptividade: critérios mensurados através dos feedbacks dados pelos usuários a respeito da ajuda que lhes foi prestada, considerando que usuários que tiveram suas respostas bem avaliadas tem tanto conhecimento técnico quanto receptividade, os quais lhes permitiram responder a dúvida satisfatoriamente. A arquitetura PHAVEA sugere classificar os usuários em níveis usando a equação a seguir, que considera os feedbacks recebidos pelo usuário para incrementar o nível dele.

$$
\cot =\left(\sum x-\sum y\right) * \mathbb{P}
$$

Onde:

$\mathrm{x} \leftarrow$ representa avaliações positivas das respostas dadas;

y $\leftarrow$ representa avaliações negativas das respostas dadas;

$\mathrm{P} \leftarrow$ representa o nível do perfil do usuário que esta pedindo ajuda (1=iniciante; 2=intermediário; $3=$ avançado).

$\mathrm{Na}$ presente pesquisa, foi decidido utilizar de fundamentos propostos pela arquitetura PHAVEA [Leite e Silveira 2010], assim foi definido que este cálculo será utilizado para cobrir os critérios de receptividade e conhecimento técnicas, sendo o usuário classificado como iniciante quando o resultado da equação for menor ou igual a três, intermediário quando o resultado da equação for maior que três e menor ou igual a seis, e avançado quando o resultado for maior que seis.

Esses valores foram escolhidos com base no número de questões que se estimava que cada usuário fizesse durante os testes do protótipo (descritos a seguir). Foi planejado (e executado) solicitar a cada usuário participante dos testes cadastrar um mínimo de três questões. Como consequência disto, era estimado que cada usuário receberia em média 3 perguntas para responder. Logo, com os valores estabelecidos para incrementar o nível, haveria a todos os usuários a possibilidade de subir, no mínimo, um nível (de iniciante para intermediário).

Além destes critérios, o critério Ferramentas configuradas em comum foi selecionado para ser também usado no processo de recomendação proposto, através do qual podem ser gerados índices de similaridade. Os professores são responsáveis, em diversas instituições, por configurar o ambiente de seus cursos, adicionando recursos e 
ferramentas do ambiente e configurando-os adequadamente para serem posteriormente utilizados pelos alunos. As ferramentas que determinado professor usa, podem ser utilizadas para encontrar o grupo de usuários similares (usuários que utilizem as mesmas ferramentas) formando as "vizinhanças" de usuários a partir destas informações. Além disso, o uso de determinada ferramenta, pode representar que o usuário que a utiliza possui conhecimentos técnicos sobre esta, complementando o critério anterior, apontado pelos usuários. Assim, decidiu-se incluir este critério ao processo de formação do par, especialmente considerando-se o trabalho de Leite e Silveira (2011) que mostrou que usuários com perfil de professor têm mais dificuldades do uso destes ambientes que outros tipos de usuários. Além disso, os resultados obtidos por esta survey confirmaram isso: dos usuários que reportaram que frequentemente possuem dúvidas sobre o uso do ambiente, $67 \%$ deles tinham perfil de professor.

Tendo isto em mente, e sabendo que são os usuários com este perfil os responsáveis pela configuração do ambiente, foi decidido usar as ferramentas (recursos e atividades) configuradas por eles, no ambiente, como critério através do qual serão traçados os índices de similaridade entre usuários que serão usados para se gerar a recomendação. Esta similaridade será mensurada utilizando a equação de Correlação de Pearson [Torres 2004][Cazella 2006]. O calculo de correlação de Pearson utiliza as avaliações feitas pelos usuários a itens que estes tenham consumido, para com base nisso, medir a similaridade dentre dois usuários. Assim, cada usuário do sistema de ajuda em pares proposto deverá fornecer ao sistema informações a respeito das ferramentas que utiliza no ambiente e avaliar seus conhecimentos sobre cada uma, partindo da ideia que usuários que tem perfil de uso similares no ambiente (utilizam os mesmos recursos) vão conseguir se ajudar.

Assim é definido que o processo de formação do par que irá auxiliar outro usuário com sua dúvida, primeiramente deverá buscar pelos usuários similares dentre o grupo de usuários (usuários com ferramentas configuradas em comum). Este grupo de usuários pode ser:

- Todos os usuários, se o usuário que cadastrou a dúvida não tenha informado à qual recurso/atividade a dúvida estaria relacionada.

- Todos os usuários com aptidão no recurso/atividade da dúvida, se o usuário que cadastrou a dúvida tenha informado à qual recurso/atividade a dúvida estaria relacionada.

Após, caso exista mais de um usuário similar, o sistema procura, dentre eles, os usuários que estejam no mesmo nível que ele (nível calculado com base nos feedbacks recebidos pelas respostas dadas). Caso exista mais de um usuário similar, e no mesmo nível, o sistema busca dentre eles o usuário que usa o ambiente a mais tempo (tempo de uso informado pelo usuário ao se cadastrar no sistema de ajuda). Caso seja encontrado mais de um usuário nesta última seleção, então a escolha do usuário dentre eles dá-se de forma randômica.

Se em alguma das etapas de busca não forem encontrados usuários, então o sistema "pula" para a próxima etapa. E se em alguma das etapas, independente de qual, somente um usuário for encontrado, este será o usuário para o qual a dúvida será encaminhada, e o restante do processo de busca será ignorado. 
Para obter os dados necessários à análise e avaliação do processo proposto, um protótipo de sistema de ajuda em pares para ambientes colaborativos de ensino foi criado. Os detalhes sobre este e seus resultados, serão apresentados a seguir.

\subsection{Protótipo}

Embora a ideia de ajuda em pares normalmente aborde sistemas de comunicação síncrona, com perguntas e respostas geradas em tempo real, nessa pesquisa foi escolhido desenvolver um sistema assíncrono para permitir uma maior flexibilidade de acesso aos usuários.

O protótipo foi disponibilizado somente para usuários com perfil professor no ambiente colaborativo de ensino Moodle ${ }^{2}$. Essas escolhas foram feitas para tentar dar uma maior objetividade ao sistema proposto, focando-se inicialmente em um público mais restrito.

O protótipo foi desenvolvido na linguagem Java, sendo composto de 4 tabelas, desenvolvidas no banco de dados MySql. O protótipo disponibilizava aos usuários as seguintes páginas:

- Meu Moodle: a página Meu Moodle era o local aonde os usuários podiam (e deveriam) informar os recursos e atividades do ambiente que utilizavam ou já haviam utilizado, e o seu nível de aptidão para cada um deles, nível que ia 0 a 4 , sendo que 0 representava não conhecer o recurso/atividade e 4 representava conhecer, saber configurar e usar com frequência.

- Cadastro de dúvidas: nesta página os usuários podiam cadastrar suas dúvidas, informando ou não o recurso/atividade ao qual esta se relaciona.

- FAQ: nesta página, as dúvidas cadastradas por outros usuários, já respondidas e que tiveram essas respostas avaliadas positivamente, eram apresentadas.

- Outras páginas: além das páginas principais citadas, o protótipo também possuía páginas através da quais o usuário podia responder as dúvidas que lhes foram encaminhadas e avaliar as respostas que foram dadas as suas dúvidas. Sobre a resposta de dúvidas, os usuários poderiam através da interface informar que não sabiam responder a determinada questão, e assim ela era encaminhada a um novo usuário. De forma semelhante, sobre a avaliação de respostas, os usuários poderiam avaliar a resposta negativamente (ou seja, como não satisfatória), e assim, a dúvida era encaminhada para um novo usuário.

O protótipo foi disponibilizado para uso, para que insumos pudessem ser produzidos de forma a possibilitar a análise do processo de recomendação para sistemas de ajuda em pares. Os detalhes sobre seu uso e os resultados obtidos são apresentados nas seções seguintes.

\footnotetext{
${ }^{2} \mathrm{O}$ ambiente Moodle foi escolhido por ser o ambiente utilizado na instituição de ensino onde a presente pesquisa foi elaborada e por essa razão este era o ambiente com que o autor da pesquisa tinha mais conhecimento, tornando mais fácil o processo de identificação dos recursos e atividades disponíveis no ambiente que deveriam ser mapeadas no protótipo.
} 


\subsection{Avaliação do processo de recomendação}

Para analisar e avaliar o processo proposto, o protótipo criado foi disponibilizado para uso durante duas semanas.

A amostra de usuários era composta de 33 professores, selecionados por conveniência, que utilizavam ou já haviam utilizado o Moodle como apoio a suas atividades de ensino, com uma média de 4,5 anos de experiência de uso, e conheciam e utilizavam uma média de 11 recursos e/ou atividades disponíveis no ambiente.

\section{Dúvidas Cadastradas}

Dos 33 usuários que se cadastraram no protótipo, 25 cadastraram dúvidas no sistema, tendo essas dúvidas sido encaminhadas para 22 usuários. Durante o período de uso do protótipo, foram cadastradas 72 dúvidas, sendo que destas, 16 não foram respondidas.

\section{Análise}

Após a pergunta ser respondida, sua resposta era enviada ao demandante da dúvida, para que este a avaliasse, informando se ela havia sido ou não satisfatória. Das 56 dúvidas respondidas, 42 foram qualificadas positivamente (respostas satisfatórias), 13 qualificadas negativamente (respostas não satisfatórias) e uma não foi qualificada pelo usuário demandante da dúvida.

As respostas que eram negativamente qualificadas pelo demandante da dúvida eram encaminhadas para um novo usuário para uma nova tentativa de resposta. Nesta segunda tentativa, 5 das 13 dúvidas não foram respondidas, 6 delas foram respondidas e positivamente qualificadas, 1 foi respondida e negativamente qualificada e uma foi respondida e não qualificada. A dúvida que foi negativamente qualificada na segunda resposta foi submetida a uma terceira tentativa de resposta, no entanto não foi respondida antes do fim do período de uso do protótipo.

Desta forma, ao final do período de uso do protótipo, das 72 dúvidas registradas, 48 foram respondidas e finalizadas com uma avaliação positiva da resposta, 22 não foram respondidas e duas foram respondidas, mas não qualificadas.

Cabe ressaltar que, durante o período de uso do protótipo, o algoritmo responsável por procurar o usuário adequado ${ }^{3}$ foi executado pelo sistema 115 vezes. Destes, em apenas 12 casos o algoritmo não conseguiu encontrar usuários similares ao demandante da dúvida, tendo nesses casos, sido usado apenas o nível do usuário e o tempo de uso que o mesmo possuía no ambiente como base para encontrar o "par". Já o caminho completo ${ }^{4}$ de busca por um "par" foi executado em 92 casos.

\subsection{Discussão}

Juntamente a usuários de ambientes colaborativos de ensino que utilizaram o protótipo pelo período de duas semanas, foi possível verificar que mesmo com um número

\footnotetext{
${ }^{3} \mathrm{O}$ algoritmo pesquisava o usuário conforme os critérios levantados e apresentados nas seções anteriores.

${ }^{4} \mathrm{O}$ caminho completo ocorria quando, dentre os diversos usuários similares encontrados, era feita uma filtragem, selecionando os usuários que estavam no mesmo nível do usuário demandante da dúvida e, dentre esse grupo, era selecionado o usuário com mais tempo de uso do ambiente.
} 
relativamente baixo de usuários para um sistema de recomendação (33 participantes), $75 \%$ das dúvidas respondidas foram qualificadas positivamente (resposta satisfatória). Durante seu uso a similaridade foi usada como critério para formação dos pares em cerca de $90 \%$ dos casos e das dúvidas que foram respondidas e que foram qualificadas positivamente, mais de $80 \%$ tiveram seus pares gerados através de similaridade, incluindo aquelas que necessitaram passar por mais de um usuário para serem respondidas. Isso mostra que há eficácia do uso de técnicas de recomendação agregadas ao processo de seleção de pares de sistemas de ajuda em pares.

Sobre o protótipo, os usuários apontaram como pontos fortes a clareza e a simplicidade deste, assim como, a possibilidade que tiveram de esclarecer dúvidas que possuíam sobre o uso do ambiente, sendo sugerido, por eles, que o sistema fosse disponibilizado para uso no Moodle através de um plugin que pudesse ser utilizado em suas atividades diárias de forma a auxiliar na utilização do ambiente.

No entanto, foi ressaltado por um dos usuários que "depende da vontade dos demais usuários para que o sistema fique o mínimo utilizável”. Os dados obtidos pelo uso do protótipo comprovam isso. Foi observado que vários usuários em algum momento interromperam suas interações com o sistema, não voltando mais ao mesmo para responder dúvidas, ou avaliar as respostas que foram informadas as dúvidas que eles cadastraram, tendo o sistema, ao final do período de uso, nove usuários com atividades pendentes.

Essa baixa participação pode ter como causador a assincronicidade do sistema. Esta assincronicidade mostrou tanto vantagens quanto desvantagens. Por um lado, foi oferecido aos usuários um sistema on-line que eles poderiam vir a acessar a qualquer momento, e de qualquer lugar durante o período do experimento, facilitando seu acesso e suas participações no experimento. No entanto, por não ser um sistema síncrono, muitas dúvidas acabaram por ficar sem resposta, o que causou um prejuízo tanto para o sistema, que não cumpriu com seu objetivo nestes casos, quanto para alguns usuários, que foram frustrados ao aguardar por uma resposta que não veio.

Um dos participantes também apontou que apesar da efetividade do sistema, ele utilizaria inicialmente de outros mecanismos para solucionar a dúvida (sites de busca) por ser uma forma mais rápida: "sempre buscaria na internet (Google) por soluções as minhas duvidas por ser mais rápido de conseguir. Apenas utilizaria este sistema caso não consiga resultados na primeira opção e no sistema de ajuda, esperando que ali estejam disponíveis pessoas especialistas para responder minha dúvida". Em contra partida, outros usuários gostaram da possibilidade de poder ter a dúvida respondida sem necessidade de sair do ambiente. Segundo um deles: "Eu adoraria ter ele integrado com o Moodle para quando eu estiver tentando fazer algo e não souber, poder gerar imediatamente a pergunta sem ter que sair do Moodle em si”.

Dentre os problemas encontrados no sistema, um dos mais críticos foi identificado através do recurso que teve o segundo maior número de dúvidas cadastradas. O recurso "Conteúdo de pacote IMS" teve 10 dúvidas cadastradas, no entanto nenhum dos usuários cadastrados no sistema sabia informou saber utiliza-lo. Desta forma, essas dúvidas ficaram "circulando" pelo sistema, passando por usuários que informavam não saber responde-las, e usuários que tentavam responde-las, mas sem sucesso. Assim, algumas dessas dúvidas tiveram um longo percurso no sistema, tendo 
passado por até cinco usuários diferentes, e não tendo obtido uma resposta satisfatória ao final de período de uso.

\section{Considerações finais}

Os sistemas de recomendação são considerados ferramentas importantes para auxiliar usuários a encontrar itens que lhes interessem, sendo esses itens produtos, conteúdos ou até mesmo, pessoas, sendo que este último caracteriza estes sistemas como sistemas de combinação social.

A pesquisa realizada verificou que ambientes colaborativos de ensino formam um "terreno fértil" para o surgimento de dúvidas sobre seu uso, devido, principalmente, a diversidade de perfis de usuários que os utilizam e também a diversidade de recursos personalizáveis que eles possuem. Em especial, usuários com perfil professor, que são responsáveis por configurar tais recursos, são o grupo de usuários mais suscetível a ter dificuldades na utilização do ambiente. Ainda, em grande parte dos casos, os usuários destes ambientes recorrem a outros usuários na tentativa de sanar suas dúvidas. Assim, a presente pesquisa objetivou prover apoio à formação de pares em sistemas de ajuda em pares, visando qualificar a ajuda oferecida pelo sistema de ajuda do ambiente, utilizando para isso um sistema de recomendação, alimentado por informações referentes ao uso de ambientes colaborativo de ensino e informações a respeito de seus usuários. Pretendeuse com isso tentar qualificar a compreensão destes usuários a respeito do ambiente em que estão inseridos.

Conforme descrito anteriormente os dados gerados pelo uso do protótipo mostram eficácia no uso de técnicas de recomendação agregadas ao processo de seleção de pares de sistemas de ajuda em pares. No entanto, não é possível afirmar que houve qualificação da ajuda se comparado a um sistema de ajuda em pares que não use tais técnicas, pois não foi realizado um grupo de controle, sem o uso de recomendação no processo de formação dos pares para que tal verificação fosse possível.

Sendo assim, este estudo apontou como sendo necessária a continuação da investigação do tema desta pesquisa, aplicando ao processo e ao protótipo os refinamentos necessários e submetendo-o para uso por um tempo mais prolongado, por meio do qual se conseguiria subsídios para o aprofundamento das discussões associadas, alimentando, também, futuras pesquisas nesta linha.

É importante, também, analisar questões a cerca da assincronicidade que o sistema atual dispõe, e verificar alternativas que combinem a flexibilidade do sistema assíncrono, que permite que usuários a qualquer momento acessem o sistema e forneçam respostas às dúvidas cadastradas, com a dinamicidade do sistema síncrono que é capaz de dar ao usuário respostas rápidas que os ajudem a sanar suas dúvidas.

Agregar aos algoritmos de recomendação a capacidade de aprendizado e possibilitar aos usuários a qualificação parcial da dúvida, encaminhando ela para novos usuários (ou de forma aberta a todos os usuários, através de um fórum) para ter a resposta complementada, são, também, refinamentos a serem feitos no processo visando sua melhoria. No entanto, é importante que em pesquisa futuras, grupos de controle sejam criados, para que seja possível comprovar a eficácia do uso de técnicas de recomendação integradas a sistemas de ajuda em pares. 


\section{Referências}

Barcellos, C. D.; Musa, D. L.; Brandão, A. L.; Warpechowski, M. "Sistemas de recomendação acadêmico para apoio a aprendizagem". RENOTE - Revistas Novas Tecnologias na Educação, vol. 5-2, Dez 2007, 10p.

Baqueta, J. J.; Teixeira, M. F.; Camargo, T. J.; Boscarioli, C. "O Moodle na pósgraduação latu sensu: uma experiência de avaliação de usabilidade”. In: Encontro Paranaense de Computação, 2011, pp. 110-119.

Cazella, S. C. "Aplicando a relevância da opinião de usuários em sistemas de recomendação para pesquisadores". Tese de Doutorado, Programa de Pós-Graduação em Ciência da Computação, UFRGS, 2006, 180p.

Cazella, S. C.; Reategui, E. B.; Nunes, M. A. "A ciência da opinião: estado da arte em sistemas de recomendação”. In: XXX Congresso da SBC Jornada de Atualização da Informática, 2010, 52p.

Damon, H.; Kamvar, S. D. "The anatomy of a large-scale social search engine”. In: 19th International conference on World Wide Web, 2010, pp 431-440.

Joseph, M. S. "Helping users help themselves", In: 8th Annual ACM Conference on User Services, 1980, pp. 108-110.

Kumar, V. S. “An instrument for providing formative feedback to novice programmers". In: Annual Meeting of American Educational Research Association, 2004, p. 72.

Leite, L. L.; Silveira, M. S. "Phavea: um arquitetura de peer help para o Moodle". In: XXXVII Seminário Integrado de Software e Hardware, 2010, pp. 478-488.

Motta, C. L. R.; Garcia A. C. B.; Vivacqua, A. S.; Santoro, F. M.; Sampaio, J. O. "Sistemas de recomendação". In: Pimentel, M.; Fuks, H. "Sistemas colaborativos". Rio de Janeiro: Elsevier, 2011, 416p.

Müller, L.; Silveira, M. S. "E agora, quem poderá me ajudar? Utilizando sistemas de recomendação para otimização do processo de busca por auxílio em ambientes colaborativos de ensino". In: $23^{\circ}$ Simpósio Brasileiro de Informática na Educação, 2012, 10p.

Ricci, F.; Rokach, L.; Shapira, B.; Kantor, P. B. "Recommender systems handbook". New York: Springer, 2010, 872p.

Spool, J.; Scalon, T. "Making online information usable". HyperViews, vol. 3-4, Nov 1996, pp. 5-7.

Torres, R. "Personalização na internet: como descobrir hábitos de consume de seus clientes, fidelizá-los e aumentar o lucro de seu negócio". Novatec Editora, 2004, $159 \mathrm{p}$.

Vouligny, L.; Robert, J. "Online help system design based on the situated action theory”. In: 2005 Latin American conference on Human-computer interaction, 2005, pp 64-75. 